PROCEEDINGS OF THE

AMERICAN MATHEMATICAL SOCIETY

Volume 129, Number 10, Pages 2845-2852

S 0002-9939(01)05908-1

Article electronically published on March 29, 2001

\title{
AN ANALOGUE OF HAJÓS' THEOREM FOR THE CIRCULAR CHROMATIC NUMBER
}

\author{
XUDING ZHU
}

(Communicated by John R. Stembridge)

\begin{abstract}
This paper designs a set of graph operations and proves that starting from $G_{d}^{k}$, by repeatedly applying these operations, one can construct all graphs $G$ with $\chi_{c}(G) \geq k / d$ (for $k / d \geq 3$ ). This can be viewed as an analogue of Hajós' Theorem for the circular chromatic number.
\end{abstract}

\section{INTRODUCTION}

The circular chromatic number (also known as the star chromatic number) of a graph is a natural generalization of the chromatic number of a graph. Given two integers $k, d$ such that $k \geq d$, a $(k, d)$-coloring of a graph $G$ is a coloring $\phi$ of the vertices of $G$ with colors $0,1,2, \cdots, k-1$ such that for any two adjacent vertices $x$ and $y$ of $G$, we have $d \leq|\phi(x)-\phi(y)| \leq k-d$. The circular chromatic number $\chi_{c}(G)$ of $G$ is the infimum of the ratio $k / d$ for which there exists a $(k, d)$-coloring of $G$. An equivalent definition of $\chi_{c}(G)$ is as follows: Suppose $r \geq 1$ is a real number. Let $C^{r}$ be a circle in the Euclidean plane of circumference $r$. For a graph $G=(V, E)$, an $r$-circular coloring $f$ of $G$ is a mapping which maps each vertex $x$ of $G$ to a unit length arc $f(x)$ of $C^{r}$ such that for every edge $x y$ of $G, f(x) \cap f(y)=\varnothing$. The circular chromatic number $\chi_{c}(G)$ of $G$ is equal to the infimum of those $r$ for which $G$ has an $r$-circular coloring. (See [12, 17 for the proof of the equivalence.) We shall use this definition of $\chi_{c}(G)$ in this paper. It was shown in 11] (also see 2, 12 for combinatorial proofs) that for finite graphs, the infimum in the definition is always attained, and hence it can be replaced by minimum.

Note that a $(k, 1)$-coloring of a graph $G$ is just an ordinary $k$-coloring of $G$. This implies that $\chi_{c}(G) \leq \chi(G)$ for any graph $G$. On the other hand, it was shown in 2, 11 that $\chi_{c}(G)>\chi(G)-1$. Therefore $\chi(G)-1<\chi_{c}(G) \leq \chi(G)$. So $\chi(G)=\left\lceil\chi_{c}(G)\right\rceil$. In this sense, $\chi_{c}(G)$ is a refinement of $\chi(G)$, and $\chi(G)$ is an approximation of $\chi_{c}(G)$.

Many questions that have been studied for the chromatic number can be studied in the framework of circular chromatic number. Quite a few classical results concerning the chromatic number of graphs have been generalized to results concerning the circular chromatic number. For example, it was shown in [13] that for

Received by the editors November 16, 1999 and, in revised form, February 21, 2000.

2000 Mathematics Subject Classification. Primary 05C15.

This research was partially supported by the National Science Council under grant NSC 892115-M-110-003. 
any $r \geq 2$ there are graphs $G$ of arbitrary large girth and with $\chi_{c}(G)=r$ (see [17] for a constructive proof). As a complement to the Four Colour Theorem, it was shown in [7, 15, 16] that a rational number $r$ is the circular chromatic number of a non-trivial planar graph if and only if $2 \leq r \leq 4$.

In this paper, we present an analogue of Hajós' theorem for the circular chromatic number. To be precise, we seek a recursive construction method that constructs all graphs $G$ with $\chi_{c}(G) \geq r$ by following some simple rules. We present such a construction method for any rational $r \geq 3$. The problem of finding an analogue of Hajós' theorem for the circular chromatic number is posed in [14, 17]. The first attempt at this problem is given in [14, where interesting examples of graph with given circular chromatic number was constructed (although an analogue of Hajós' theorem was not found). In this paper, we use three operations to replace Hajós' sum. Among these operations, Operation 1 has already been used in [14].

\section{Operations that do NOT DECREASE CIRCUlar CHROMATIC NUMBER}

Suppose $G, G^{\prime}$ are graphs, and $e=x y, e^{\prime}=x^{\prime} y^{\prime}$ are edges of $G, G^{\prime}$ respectively. The Hajós' sum of $G$ and $G^{\prime}$ (with respect to $e$ and $e^{\prime}$ ) is the graph obtained from the disjoint union of $G$ and $G^{\prime}$ by deleting $e$ and $e^{\prime}$, identifying $x$ and $x^{\prime}$, and adding an edge that connects $y$ and $y^{\prime}$.

Let $\mathcal{G}_{n}$ denote the class of graphs with chromatic number at least $n$. Hajós [5] proved the following theorem:

Theorem 1. The class $\mathcal{G}_{n}$ is the minimal class of graphs which contains $K_{n}$ and is closed under the following operations:

1. adding vertices and edges;

2. identifying non-adjacent vertices;

3. taking Hajós' sum of two graphs.

Given a rational number $r=k / d \geq 2($ with $\operatorname{gcd}(k, d)=1)$. The graph $G_{k}^{d}$ has vertices $0,1, \cdots, k-1$, and $i j$ is an edge if and only if $d \leq|i-j| \leq k-$ $d$. From the point of view of graph homomorphisms, the graphs $G_{k}^{d}$ play the same role in the investigation of circular chromatic number as those played by the complete graphs in the investigation of chromatic number: A graph $G$ has circular chromatic number at most $k / d$ if and only if $G$ admits a homomorphism to $G_{k}^{d}$ (i.e., an edge-preserving mapping from the vertex set of $G$ to the vertex set of $\left.G_{d}^{k}\right)$. Thus an analogue of Hajós' theorem for the circular chromatic number would construct all graphs of circular chromatic number at least $k / d$ from the graph $G_{k}^{d}$ by a set of simple rules. It is easy to see that the operations of adding vertices and edges, and identifying nonadjacent vertices do not decrease the circular chromatic number of a graph. However, the operation of taking Hajós' sum may decrease the circular chromatic number. For example, the Hajós' sum of two triangles produces a pentagon, while the triangle has circular chromatic number 3 and the pentagon has circular chromatic number $5 / 2$.

In this section, we shall present 3 operations that do not decrease the circular chromatic number. These operations will be used to replace the Hajós' sum to construct all graphs of circular chromatic number at least $k / d$ from copies of $G_{d}^{k}$.

Operation 1. Given $2 k+1(k \geq 1)$ graphs $G_{0}, G_{1}, G_{2}, \cdots, G_{2 k}$. For $i=0,1,2, \cdots$, $2 k$, let $e_{i}=x_{i} y_{i}$ be an edge of $G_{i}$. Let $C_{2 k+1}=\left(c_{0}, c_{1}, \cdots, c_{2 k}\right)$ be a cycle of length $2 k+1$. Construct a new graph from the disjoint union of $G_{0}, G_{1}, G_{2}, \cdots, G_{2 k}, C_{2 k+1}$ 
as follows:

- delete the edges $e_{i}$, for $i=0,1,2, \cdots, 2 k$,

- identify all the $x_{i}$ 's into a single vertex and name it $x$,

- identify $y_{i}$ and $c_{i}$ for $i=0,1,2, \cdots, 2 k$.

We shall denote the resulting graph by $S_{1}\left(G_{0}, e_{0}, G_{1}, e_{1}, G_{2}, e_{2}, \cdots, G_{2 k}, e_{2 k}\right)$, or simply by $S_{1}$ if the graphs $G_{i}$ and edges $e_{i}$ are clear from the context.

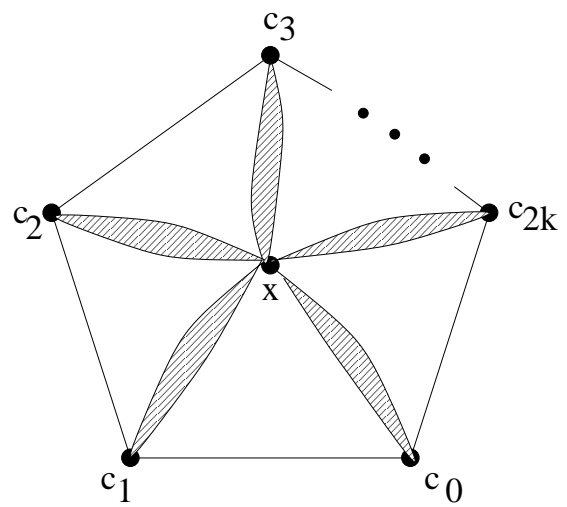

FIGURE 1. Operation 1 (each shaded area represents a $G_{i}-e_{i}$ )

Lemma 1. If $k \geq 1$ and $\chi_{c}\left(G_{i}\right) \geq r \geq 2$ for $i=0,1, \cdots, 2 k$, then $\chi_{c}\left(S_{1}\right) \geq r$.

Proof. We refer to Figure 1 for the names of the vertices. Assume to the contrary that for some $r^{\prime}<r$, there is an $r^{\prime}$-circular coloring of $S_{1}$. Since $\chi_{c}\left(G_{i}\right) \geq r$ for each $i$, we conclude that $f(x) \cap f\left(c_{i}\right) \neq \varnothing$. Assume that the two end points of $f(x)$ are $p_{1}$ and $p_{2}$. Since $f\left(c_{1}\right) \cap f\left(c_{0}\right)=\varnothing$, we conclude that one of $f\left(c_{0}\right), f\left(c_{1}\right)$ contains $p_{1}$ and the other contains $p_{2}$. Without loss of generality, we assume that $p_{1} \in f\left(c_{1}\right)$ and $p_{2} \in f\left(c_{0}\right)$. Since $f\left(c_{1}\right) \cap f\left(c_{2}\right)=\varnothing$, we conclude that $p_{2} \in f\left(c_{2}\right)$. By induction, we conclude that $p_{2} \in f\left(c_{2 j}\right)$ and $p_{1} \in f\left(c_{2 j-1}\right)$ for any $j \geq 1$. In particular, $p_{2} \in f\left(c_{2 k}\right)$. So $f\left(c_{0}\right) \cap f\left(c_{2 k}\right) \neq \varnothing$, contrary to the assumption that $f$ is an $r^{\prime}$-coloring of $S_{1}$.

Operation 2. Given $n$ graphs $G_{0}, G_{1}, G_{2}, \cdots, G_{n-1}$. For $i=0,1,2, \cdots, n-1$, let $e_{i}=x_{i} y_{i}$ be an edge of $G_{i}$. Construct a new graph from the disjoint union of $G_{0}, G_{1}, G_{2}, \cdots, G_{n-1}$ as follows:

- delete the edges $e_{i}$, for $i=0,1,2, \cdots, n-1$,

- identify $y_{i}$ with $x_{i+1}$, for $i=0,1,2, \cdots, n-1$ (here and below the sum in the indices are modulo $n$ ),

- add edges to connect $x_{i}$ and $x_{i+2}$ for $i=0,1,2, \cdots, n-1$,

- add a vertex $u$ and connect $u$ to $x_{i}$ for $i=0,1,2, \cdots, n-1$.

We shall denote the resulting graph by $S_{2}\left(G_{0}, e_{0}, G_{1}, e_{1}, G_{2}, e_{2}, \cdots, G_{n-1}, e_{n-1}\right)$, or simply by $S_{2}$ if the graphs $G_{i}$ and edges $e_{i}$ are clear from the context.

Lemma 2. If $n \geq 4$ and $\chi_{c}\left(G_{i}\right) \geq r \geq 2$ for $i=0,1, \cdots, n-1$, then $\chi_{c}\left(S_{2}\right) \geq r$. 


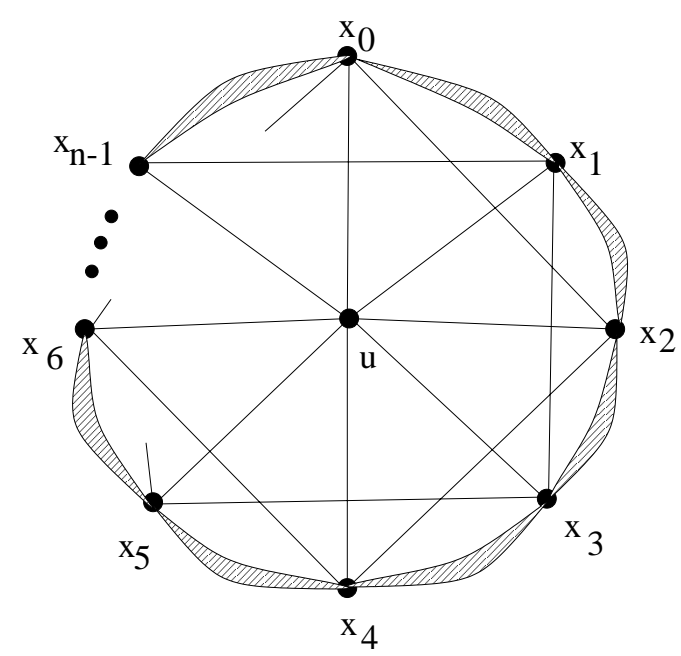

FiguRE 2. Operation 2 (each of the shaded area represents a $G_{i}-e_{i}$ )

Proof. Again we refer to Figure 2 for the names of the vertices. Assume to the contrary that there is an $r^{\prime}<r$ and an $r^{\prime}$-circular coloring $f$ of $S_{2}$. Since $\chi_{c}\left(G_{i}\right) \geq$ $r>r^{\prime}$, it follows that for $i=0,1, \cdots, n-1, f\left(x_{i}\right) \cap f\left(x_{i+1}\right) \neq \varnothing$. On the other hand $f\left(x_{i}\right) \cap f\left(x_{i+2}\right)=\varnothing$ (for $i=0,1,2, \cdots, n-1$ ). It follows that the relative positions of the arcs $f\left(x_{i}\right)$ must be as depicted in Figure 3.

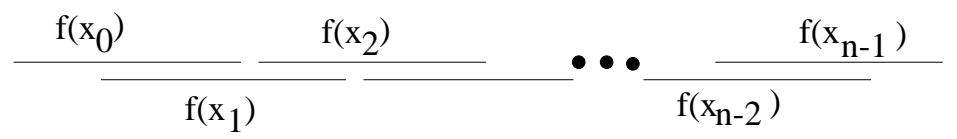

Figure 3. Relative positions of $f\left(x_{i}\right)$ on $C^{r^{\prime}}$

However, $f\left(x_{n-1}\right)$ must intersect $f\left(x_{0}\right)$. The only possibility is that $f\left(x_{n-1}\right)$ goes around the circle $C^{r^{\prime}}$ to "meet" $f\left(x_{0}\right)$ at the left end point. Therefore the union

$$
f\left(x_{0}\right) \cup f\left(x_{1}\right) \cup f\left(x_{2}\right) \cup \cdots \cup f\left(x_{n-1}\right)
$$

covers all the circle $C^{r^{\prime}}$. Now $f(u)$ is disjoint from each of the $\operatorname{arcs} f\left(x_{i}\right)$. This is an obvious contradiction.

Operation 3. Given seven graphs $G_{1}, G_{2}, \cdots, G_{7}$. Let $P_{4}$ be the path with five vertices $p_{1}, p_{2}, \cdots, p_{5}$. For $i=1,2, \cdots, 7$, let $e_{i}=x_{i} y_{i}$ be an edge of $G_{i}$. Construct a new graph from the disjoint union of $G_{1}, G_{2}, \cdots, G_{7}$ and $P_{4}$ as follows:

- delete the edges $e_{i}$, for $i=1,2, \cdots, 7$,

- identify $y_{1}, y_{2}, y_{3}, y_{4}, y_{5}$ into a single vertex, and name it $y$, and identify $x_{i}$ with $p_{i}$ for $i=1,2, \cdots, 5$, and name it $x_{i}$,

- identify $x_{6}$ with $x_{2}, y_{6}$ with $x_{5}, x_{7}$ with $x_{1}, y_{7}$ with $x_{4}$.

We shall denote the resulting graph by $S_{3}\left(G_{1}, e_{1}, G_{2}, e_{2}, \cdots, G_{7}, e_{7}\right)$, or simply by $S_{3}$ if the graphs $G_{i}$ and edges $e_{i}$ are clear from the context. 


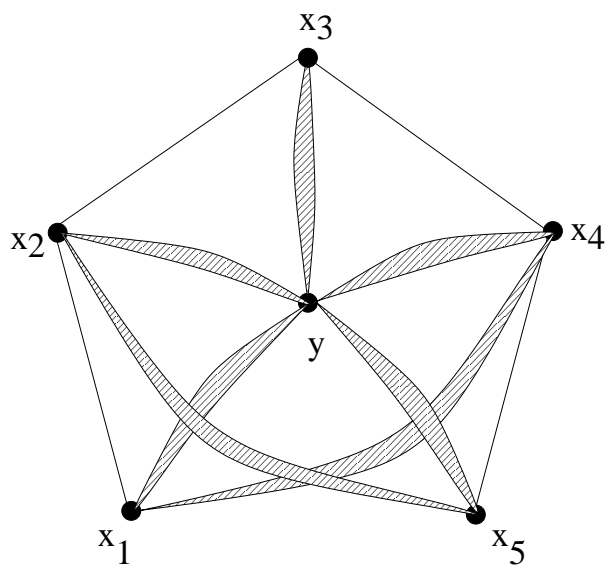

Figure 4. Operation 3 (each shaded area represents a $G_{i}-e_{i}$ )

Lemma 3. If $r \geq 3$ and $G_{1}, G_{2}, \cdots, G_{7}$ are graphs with circular chromatic number at least $r$, then $\chi_{c}\left(S_{3}\right) \geq r$.

Proof. Assume to the contrary that there is an $r^{\prime}<r$ and an $r^{\prime}$-circular coloring $f$ of $S_{3}$.

Since $\chi_{c}\left(G_{i}\right) \geq r$ for each $i, f\left(x_{i}\right) \cap f(y) \neq \varnothing$ for $i=1,2, \cdots, 5$. Assume that $f\left(x_{1}\right) \cap f(y)$ is an arc of length $\delta, f\left(x_{2}\right) \cap f(y)$ is an arc of length $\delta^{\prime}$.

We may assume that $r-r^{\prime}<\min \left\{\delta, \delta^{\prime}\right\}$. Otherwise, we choose an $\epsilon>0$ so that $0<r-r^{\prime}-\epsilon<\min \left\{\delta, \delta^{\prime}\right\}$, choose a point $p \notin f(y)$ on the circle $C^{r^{\prime}}$, then insert a segment of length $\epsilon$ at the point $p$, so we obtain a circle of length $r^{\prime}+\epsilon$. Now each arc $f(v)$ in the new circle has length 1 if $f(v)$ does not contain $p$, and has length $1+\epsilon>1$ if $f(v)$ does contain $p$. For each $v$ such that $f(v)$ contains $p$, we may fix one end point of $f(v)$ and move the other end point of $f(v)$ (in the new circle of circumference $\left.r^{\prime}+\epsilon\right)$ so that $f(v)$ has length 1 in the new circle. It is obvious that this modification gives an $\left(r^{\prime}+\epsilon\right)$-circular coloring of $S_{3}$. (Indeed, no new intersections between the arcs are introduced.) By properly choosing the end point of $f(v)$ to be fixed, we can assure that for the new $\left(r^{\prime}+\epsilon\right)$-circular coloring, the intersections $f\left(x_{1}\right) \cap f(y)$ and $f\left(x_{2}\right) \cap f(y)$ still have length $\delta$ and $\delta^{\prime}$.

Let the two end points of $f(y)$ be $p, q$. Since $f\left(x_{i}\right) \cap f\left(x_{i+1}\right)=\varnothing$ for $i=1,2,3,4$, we may assume that $p \in f\left(x_{1}\right), f\left(x_{3}\right), f\left(x_{5}\right)$ and $q \in f\left(x_{2}\right), f\left(x_{4}\right)$. Referring to Figure 4, we see that $f\left(x_{1}\right) \cap f\left(x_{4}\right) \neq \varnothing$. The intersection $f\left(x_{1}\right) \cap f\left(x_{4}\right)$ does not contain $p$ or $q$. So either $f\left(x_{1}\right) \cap f\left(x_{4}\right)$ is contained in $f(y)$ or $f\left(x_{1}\right) \cap f\left(x_{4}\right)$ is disjoint from $f(y)$.

If $f\left(x_{1}\right) \cap f\left(x_{4}\right)$ is disjoint from $f(y)$, then the union $f\left(x_{1}\right) \cup f\left(x_{4}\right) \cup f(y)$ covers the whole circle which has length $r^{\prime}$. However, since $f\left(x_{1}\right) \cap f(y)$ has length $\delta>r-r^{\prime}$, the union $f\left(x_{1}\right) \cup f\left(x_{4}\right) \cup f(y)$ has length at most $3-\delta<3-\left(r-r^{\prime}\right)<r^{\prime}$ (as $r \geq 3$ ), which is a contradiction. Therefore $f\left(x_{1}\right) \cap f\left(x_{4}\right)$ is contained in $f(y)$.

Similarly, we have $f\left(x_{2}\right) \cap f\left(x_{5}\right) \neq \varnothing$ and this implies that the intersection $f\left(x_{2}\right) \cap f\left(x_{5}\right)$ is contained in $f(y)$. But this is impossible, because $f\left(x_{2}\right)$ is disjoint from $f\left(x_{1}\right)$ and $f\left(x_{5}\right)$ is disjoint from $f\left(x_{4}\right)$. 


\section{THE MAIN RESULT}

Now we shall prove that Operations 1,2 , and 3 , together with the operation of adding vertices and edges and identifying nonadjacent vertices, enable us to construct all graphs of circular chromatic number greater than or equal to $k / d$ from copies of $G_{d}^{k}$ (for $k / d \geq 3$ ).

Theorem 2. Suppose $k / d \geq 3$. Let $\mathcal{G}_{k}^{d}$ be the set of graphs that contains $G_{k}^{d}$ and is closed under the following operation:

- adding vertices and edges;

- identifying non-adjacent vertices;

- applying Operations 1,2,3.

Then $\mathcal{G}_{k}^{d}$ consists of all graphs $G$ with $\chi_{c}(G) \geq k / d$.

It follows from Lemmas 1, 2, and 3] that all the graphs in $\mathcal{G}_{k}^{d}$ have circular chromatic number at least $k / d$. It remains to show that every graph $G$ with $\chi_{c}(G) \geq$ $r$ is contained in $\mathcal{G}_{k}^{d}$.

For this purpose, we need the following theorem, which is proved in 18 .

Theorem 3. If $G$ is a graph such that for every vertex $x$ of $G, N_{G}[x]$ induces a perfect graph, and $V(G)-N_{G}[x]$ induces a bipartite graph with no induced $P_{4}$, then $\chi_{c}(G)=\omega_{c}(G)$, where $\omega_{c}(G)$ is the minimum $k / d$ for which $G_{d}^{k}$ is a subgraph of $G$.

Proof of Theorem 2] Assume to the contrary that there is a graph $G$ with $\chi_{c}(G) \geq$ $k / d$ and $G \notin \mathcal{G}_{d}^{k}$. We may choose the graph $G$ so that by adding any edge to $G$, the resulting graph $G^{\prime}$ is no longer a counterexample to Theorem 2 Since $\chi_{c}\left(G^{\prime}\right) \geq r$, so $G^{\prime} \in \mathcal{G}_{d}^{k}$. In other words, $G \in \mathcal{G}_{d}^{k}$, but adding any edge results in a graph $G^{\prime} \in \mathcal{G}_{d}^{k}$.

Assume that $\chi_{c}(G)=k^{\prime} / d^{\prime} \geq k / d$. First we assume that $\omega_{c}(G)=k^{\prime} / d^{\prime}$. Then $G$ contains a copy of $G_{d^{\prime}}^{k^{\prime}}$. It is well known (cf. [17]) that $G_{d}^{k}$ admits a homomorphism to $G_{d^{\prime}}^{k^{\prime}}$. Hence $G_{d}^{k}$ admits a homomorphism to $G$. Let $f$ be a homomorphism from $G_{d}^{k}$ to $G$. By identifying those vertices of $G_{d}^{k}$ which are mapped to the same vertex of $G$, and by adding some vertices and edges (if necessary), we obtain a copy of $G$.

Now we assume that $\omega_{c}(G)<k^{\prime} / d^{\prime}$. Then by Theorem 3 , we know that there is a vertex $x$ of $G$ such that one of the following is true:

1. $V(G)-N_{G}[x]$ is not bipartite;

2. $V(G)-N_{G}[x]$ contains an induced $P_{4}$;

3. $N_{G}[x]$ is not a perfect graph.

We shall derive a contradiction in each of these three cases.

First assume that $V(G)-N_{G}[x]$ contains an odd cycle $C=\left(x_{0}, x_{1}, \cdots, x_{2 k}\right)$. For $i=0,1, \cdots, 2 k$, let $G_{i}$ be obtained from $G$ by adding edge $e_{i}=x x_{i}$. Then $G_{i} \in \mathcal{G}_{d}^{k}$. Let $G^{\prime}=S_{1}\left(G_{0}, e_{0}, G_{1}, e_{1}, \cdots, G_{2 k}, e_{2 k}\right)$. Then $G^{\prime}$ is as depicted in Figure 1, where each shaded area is a copy of $G$ (as each $G_{i}-e_{i}$ is a copy of $G$ ). Moreover, for each copy of $G$, the vertex $x$ in $G^{\prime}$ is also the vertex $x$ in $G$, and the vertex $c_{i}$ in $G^{\prime}$ is the vertex $x_{i}$ in $G$.

For each vertex $v$ of $G$, let $v_{i}$ be the copy of $v$ in $G_{i}$. Now we identify all the $v_{i}$ 's into a single vertex. Because the vertices $x_{0}, x_{1}, \cdots, x_{2 k}$ already induce a cycle in $G$, the resulting graph is $G$. Therefore $G \in \mathcal{G}_{d}^{k}$, contrary to our assumption.

Assume next that $V(G)-N_{G}(x)$ contains an induced path $\left(x_{1}, x_{2}, x_{3}, x_{4}, x_{5}\right)$ of length 4 . For $i=1,2,3,4,5$, let $G_{i}$ be obtained from $G$ by adding the edge 
$e_{i}=x x_{i}$. Let $G_{6}$ be the graph obtained from $G$ by adding the edge $e_{6}=x_{1} x_{4}$ and $G_{7}$ be the graph obtained from $G$ by adding the edge $e_{7}=x_{2} x_{5}$. Then $G_{i} \in \mathcal{G}_{d}^{k}$ for $i=1,2, \cdots, 7$.

Let $G^{\prime}=S_{3}\left(G_{1}, e_{1}, G_{2}, e_{2}, \cdots, G_{7}, e_{7}\right)$. Then $G^{\prime}$ is as depicted in Figure 4 , where each shaded area is a copy of $G$ (as $G_{i}-e_{i}=G$ ). Similarly as above, for each $v \in G$, we identify the different copies of $v$ in $G^{\prime}$ into a single vertex. We obtain the graph $G$. So $G \in \mathcal{G}_{d}^{k}$, contrary to our assumption.

Now we assume that $N_{G}[x]$ induces a non-perfect graph. Then the complement of $N_{G}[x]$ contains an induced cycle of length $n \geq 4$ (recall that triangulated graphs are perfect, and complements of perfect graphs are perfect).

Assume $\left(x_{0}, x_{1}, \cdots, x_{n-1}\right)$ is an induced cycle of the complement of $N_{G}[x]$ of length $n \geq 4$. Let $G_{i}$ be obtained from $G$ by adding the edge $e_{i}=x_{i} x_{i+1}$ for $i=0,1,2, \cdots, n-1$ (summation in indices modulo $n$ ). Let

$$
G^{\prime}=S_{2}\left(G_{0}, e_{0}, G_{1}, e_{1}, \cdots, G_{n-1}, e_{n-1}\right) .
$$

Then $G^{\prime}$ is as depicted in Figure 2, where each shaded area represents a copy of $G$. Identifying the different copies of the same vertex of $G$ into a single vertex, and $u$ (as in Figure 2) with the vertex $x$ of $G$, we obtain a copy of $G$. So $G \in \mathcal{G}_{d}^{k}$, contrary to our assumption. This completes the proof of Theorem 2

Two remarks. 1 . The method presented here constructs all graphs with $\chi_{c}(G) \geq$ $k / d$ from $G_{d}^{k}$ with a few rules, provided that $k / d \geq 3$. The method does not work for $2<k / d<3$. After the submission of this paper, the author found another set of graph operations which works for $2<k / d<3$. By that method, we need four more graph operations for the case $2<k / d<3$ [19].

2. A different generalization of Hajós' theorem to $H$-coloring was given by Nešetřil [8]. Given any graph $H$, a method is presented in [8] that constructs all graphs which do not admit a homomorphism to $H$, starting from a finite set of graphs. These operations are adding vertices and edges, identifying nonadjacent vertices and a "generalized" Hajós' sum. In particular, for any $k / d \geq 2$, that method construct all graphs $G$ with $\chi_{c}(G)>k / d$ (note that here it is necessary to have the strict inequality), starting with a finite set of graphs. The finite set of graphs is not known explicitly and is usually a big set.

\section{REFERENCES}

[1] H. L. Abbott and B. Zhou, The star chromatic number of a graph, J. Graph Theory 17 (1993), 349-360. MR 94g:05032

[2] J. A. Bondy and P. Hell, A note on the star chromatic number, J. Graph Theory 14 (1990), 479-482. MR 91i:05052

[3] S. Brandt, On the structure of dense triangle-free graphs, Fachbereich Mathematik und Informatik, Series A Mathematik, Preprint No. A 97-16. CMP 99:16 (English summary)

[4] D. R. Guichard, Acyclic graph coloring and the complexity of the star chromatic number, J. Graph Theory 17 (1993), 129-134. MR 94e:05105

[5] G. Hajós, Über eine Konstruktion nicht n-färbbarer Graphen, Wiss. Zeitschrift Univ. Halle, Math.-Nat. 10 (1961), 113-114.

[6] R. Hayward, Weakly triangulated graphs, J. Combin. Th. (B), 39(1985), 200-208. MR 87h:05171

[7] D. Moser, The star-chromatic number of planar graphs, J. Graph Theory 24(1997), 33-43. MR 97k:05082

[8] J. Nešetřil, Homomorphism structure of classes of graphs, Combinatorics, Probability and computing 8 (1999), 177-184. MR 2000c:05068 
[9] J. Pach, Graphs whose every independent set has a common neighbour, Discrete Math. 37(1981), 217-228. MR 84e:05094

[10] E. Steffen and X. Zhu, On the star chromatic numbers of graphs, Combinatorica, 16 (1996) 439-448. MR 97g:05084

[11] A. Vince, Star chromatic number, J. Graph Theory 12 (1988), 551-559. MR 90c:05098

[12] X. Zhu, Star chromatic numbers and products of graphs, J. Graph Theory 16 (1992), 557-569. MR 93i:05066

[13] X. Zhu, Uniquely H-colorable graphs with large girth, J. Graph Theory, 23 (1996), 33-41. MR 97e:05089

[14] X. Zhu, Graphs whose circular chromatic number equals the chromatic number, Combinatorica, 19(1)(1999), 139-149. MR 2000j:05047

[15] X. Zhu, A simple proof of Moser's theorem, J. Graph Theory, 30(1999), 19-26. MR 99g:05084

[16] X. Zhu, Planar graphs with circular chromatic numbers between 3 and 4, J. Combinatorial Theory (B), 76(1999), 170-200. MR 2000e:05072

[17] X. Zhu, Circular chromatic number, a survey, Discrete Mathematics, to appear.

[18] X. Zhu, Circular perfect graphs, manuscript, 1999.

[19] X. Zhu, Hajós' theorem for the circular chromatic number (II), in preparation.

Department of Applied Mathematics, National Sun Yat-sen University, Kaohsiung, TAIWAN 80424

E-mail address: zhu@math.nsysu.edu.tw 